\title{
Chest pain and palpitation caused by a left ventricular haemangioma in an adult
}

\author{
Qiang Fan $^{1}$, Yunfei Ling ${ }^{1}$, Qin Yang ${ }^{1}$, and Changping Gan ${ }^{1}$ \\ ${ }^{1}$ Sichuan University West China Hospital
}

August 28, 2020

\begin{abstract}
Hemangioma in heart chambers is rare, most cases with heat hemangioma without symptom. The case we presented was an extremely left ventricle hemangioma with hest pain and palpitation after exercise. The tumor was resected under cardiopulmonary bypass, histopathological analysis concluded a benign vascular tumor. Examination of the tumor revealed vascular proliferation and mucoid degeneration in matrix. Immunohistochemistry showed strong positive staining with antibodies against CD31 and CD34, which supported the vascular origin of this tumor.
\end{abstract}

\section{Chest pain and palpitation caused by a left ventricular haemangioma in an adult}

Qiang Fan ${ }^{1 *}$ MD, Yunfei Ling ${ }^{1 *} \mathrm{PhD}$, Qin Yang ${ }^{2} \mathrm{MD}$, Changping Gan ${ }^{1 \#} \mathrm{PhD}$

1 Department of Cardiovascular surgery, West China Hospital, Sichuan University

2 Department of Radiology, West China Hospital, Sichuan University

* These authors contributed equally to this work and should be considered co-first authors

\# Corresponding author information: Tel: +86 28 85422897, Fax: +86 28 85422897, E-mail: ganchangping@hotmail.com, Add: No. 37 Guo Xue Xiang, Chengdu, Sichuan 610041, People's Republic of China.

Keywords : hemangioma, left ventricle, symptomatic

Funding : none.

Abstract Hemangioma in heart chambers is rare, most cases with heat hemangioma without symptom. The case we presented was an extremely left ventricle hemangioma with hest pain and palpitation after exercise. The tumor was resected under cardiopulmonary bypass, histopathological analysis concluded a benign vascular tumor. Examination of the tumor revealed vascular proliferation and mucoid degeneration in matrix. Immunohistochemistry showed strong positive staining with antibodies against CD31 and CD34, which supported the vascular origin of this tumor.

A 22-year-old man was presented to the emergency room with chest pain and palpitation after exercise for more than one month[1]. Physical examination showed no positive signs except the heart rates was 112 beats per minute. The patient's history was unremarkable while the body temperature was 37.7 centigrade when he checked in. Laboratory parameters were unremarkable, with no elevation in his levels of high-sensitive cardiac troponin $\mathrm{T}(4.5 \mathrm{pg} / \mathrm{ml}$, reference $<14 \mathrm{pg} / \mathrm{ml})$, N-terminal pro-brain natriuretic peptide $(<5 \mathrm{ng} / \mathrm{l}$, reference $<88 \mathrm{ng} / \mathrm{l}$ ). Transesophageal echocardiography during operation revealed a strong echo oval shaped circumscribed mass measured $17 \times 15 \mathrm{~mm}$ in his left ventricle cavity with great mobility (panels A and B, Supplementary material online, video1 and 2). His cardiac valves and the size and function of left ventricle were normal. Cardiovascular magnetic resonance $(\mathrm{CMR})$ imaging, which revealed a $17 \times 15 \mathrm{~mm}$ left ventricular 
mass that significantly enhanced after injection of gadolinium enhancement (panels C and D, Supplementary material online, video3 and 4). The patient received surgical intervention under the cardiopulmonary bypass, the tumor was identified originating from left ventricle cavity with a pedicle connected to the middle section of interventricular septal. The surface of the tumor was covered by milky white colloidal substances, and red jelly-like stuff in it (panel $\mathrm{E}$ and $\mathrm{F}$ ). Histopathological analysis concluded a benign vascular tumor. Examination of the tumor revealed vascular proliferation and mucoid degeneration in matrix (panel $\mathrm{G}$ ). Cells showed strong positive staining with antibodies against CD31 (panel H) and CD34 (panel I)[2], which supported the vascular origin of this tumor. The patient recovered uneventfully and discharged 4 days after surgery.

Consent The study were approved by the relevant ethics committees, and oral informed consent was obtained for the participant.

\section{References}

[1]. Han, Y., et al., Cardiac capillary hemangioma: a case report and brief review of the literature. J Clin Ultrasound, 2014. 42(1): p. 53-6.

[2]. Hirschberg, K., et al., Incidental finding of a giant intracardiac angioma infiltrating both ventricles in a 35-year-old woman: a case report. Journal of medical case reports, 2016. 10(1): p. 94.

Figure legend Panel A and B: intraoperative transesophageal echocardiography showed a mass in left ventricle cavity, panel $\mathrm{C}$ and $\mathrm{D}$ : cardiovascular magnetic resonance imaging revealed that the mass significantly enhanced, panel E and F: surface and inner appearance of the mass, panel G: histopathological examination of the tumor, panel $\mathrm{H}$ and I: immunohistochemistry analysis of the tumor.

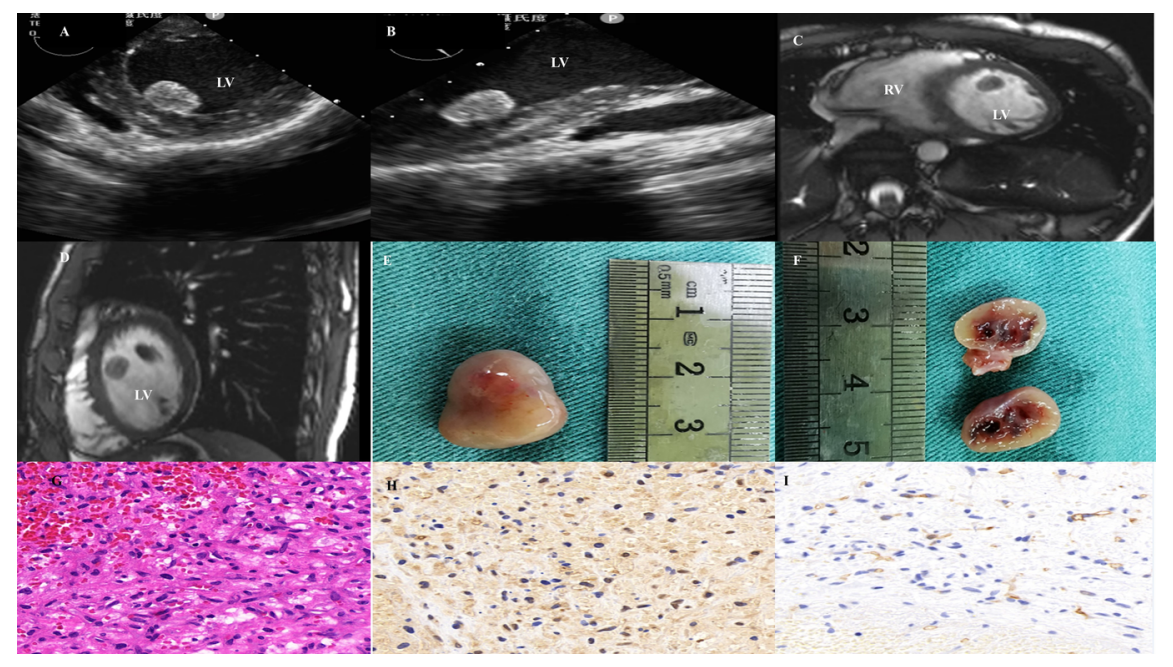

\section{Hosted file}

video 1.MP4 available at https://authorea.com/users/354240/articles/477878-chest-pain-andpalpitation-caused-by-a-left-ventricular-haemangioma-in-an-adult

\section{Hosted file}

video 2.MP4 available at https://authorea.com/users/354240/articles/477878-chest-pain-andpalpitation-caused-by-a-left-ventricular-haemangioma-in-an-adult

\section{Hosted file}

video 3.mp4 available at https://authorea.com/users/354240/articles/477878-chest-pain-andpalpitation-caused-by-a-left-ventricular-haemangioma-in-an-adult 


\section{Hosted file}

video 4.mp4 available at https://authorea.com/users/354240/articles/477878-chest-pain-andpalpitation-caused-by-a-left-ventricular-haemangioma-in-an-adult 